\title{
Peroral endoscopic myotomy for achalasia after distal gastrectomy
}

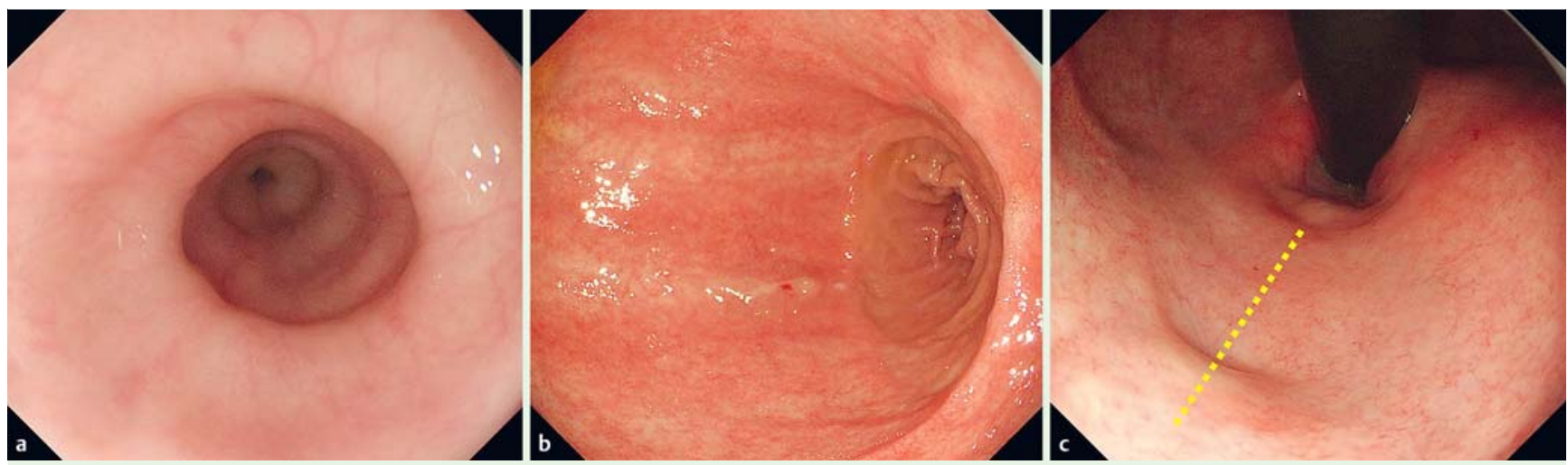

Fig. 1 Endoscopic views from a 72-year-old man with an approximately 30-year history of progressive dysphagia and regurgitation. a Dilated esophagus. b Billroth I anastomosis. c Suture line (yellow dotted line) at the lesser curvature of the remaining portion of the stomach.

Achalasia is a relatively rare esophageal motility disorder with a reported incidence of approximately 1 in 100000 worldwide [1]. The occurrence of achalasia in patients with a distal gastrectomy is rare. We present a patient with achalasia and a distal gastrectomy who was successfully treated with peroral endoscopic myotomy (POEM).

A 72-year-old man with an approximately 30-year history of progressive dysphagia and regurgitation was referred to our hospital. He had undergone a distal gastrectomy for a gastric ulcer 40 years earlier. Esophagogastroduodenoscopy showed a dilated esophagus, a Billroth I anastomosis, and a suture line along the lesser curvature ( $\bullet$ Fig. 1). Esophagography showed delayed esophageal emptying and narrowing at the lower esophageal sphincter (LES) ( $\bullet$ Fig.2). High resolution manometry demonstrated spastic contraction and impaired LES relaxation ( $\bullet$ Fig. 3 ).

We diagnosed nonsigmoid type III achalasia according to the Chicago classification and performed POEM. To avoid the suture line, the myotomy was done at the 5-o'clock position ( Fig.4). Esophagography on postoperative day 1 showed adequate passage of contrast into the remaining portion of the stomach ( $\mathbf{F i g . 5}$ ). The patient's dysphagia and regurgitation had resolved completely at follow-up 8 weeks postoperatively.

Laparoscopic Heller's myotomy is challenging in postoperative patients with achalasia because of their extensive adhesions and altered anatomy [2,3]. On the
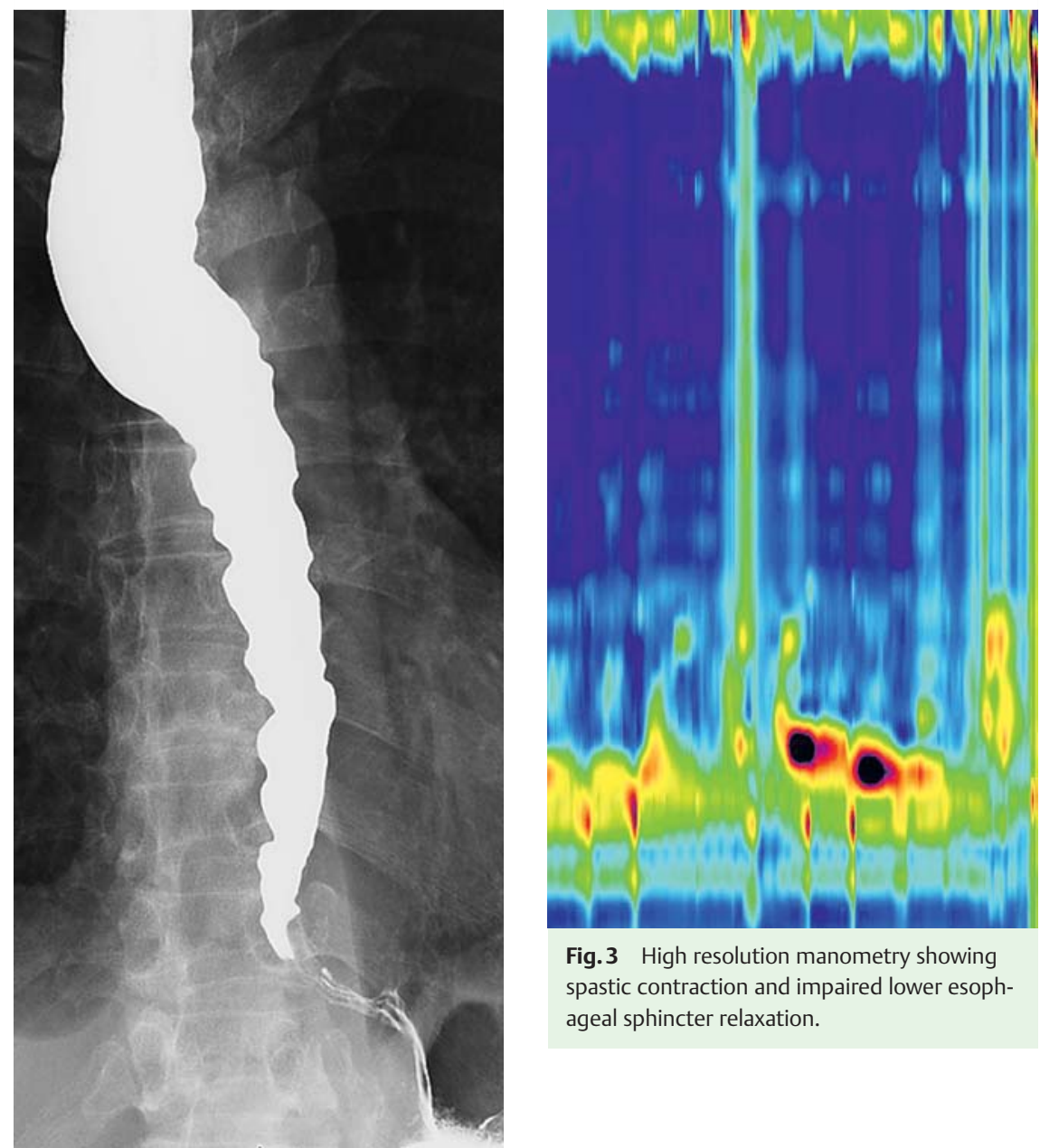

Fig. 3 High resolution manometry showing spastic contraction and impaired lower esophageal sphincter relaxation.
Fig. 2 Esophagogram showing a dilated distal esophagus with delayed emptying of the esophageal contents and tapering at the lower esophageal sphincter. 


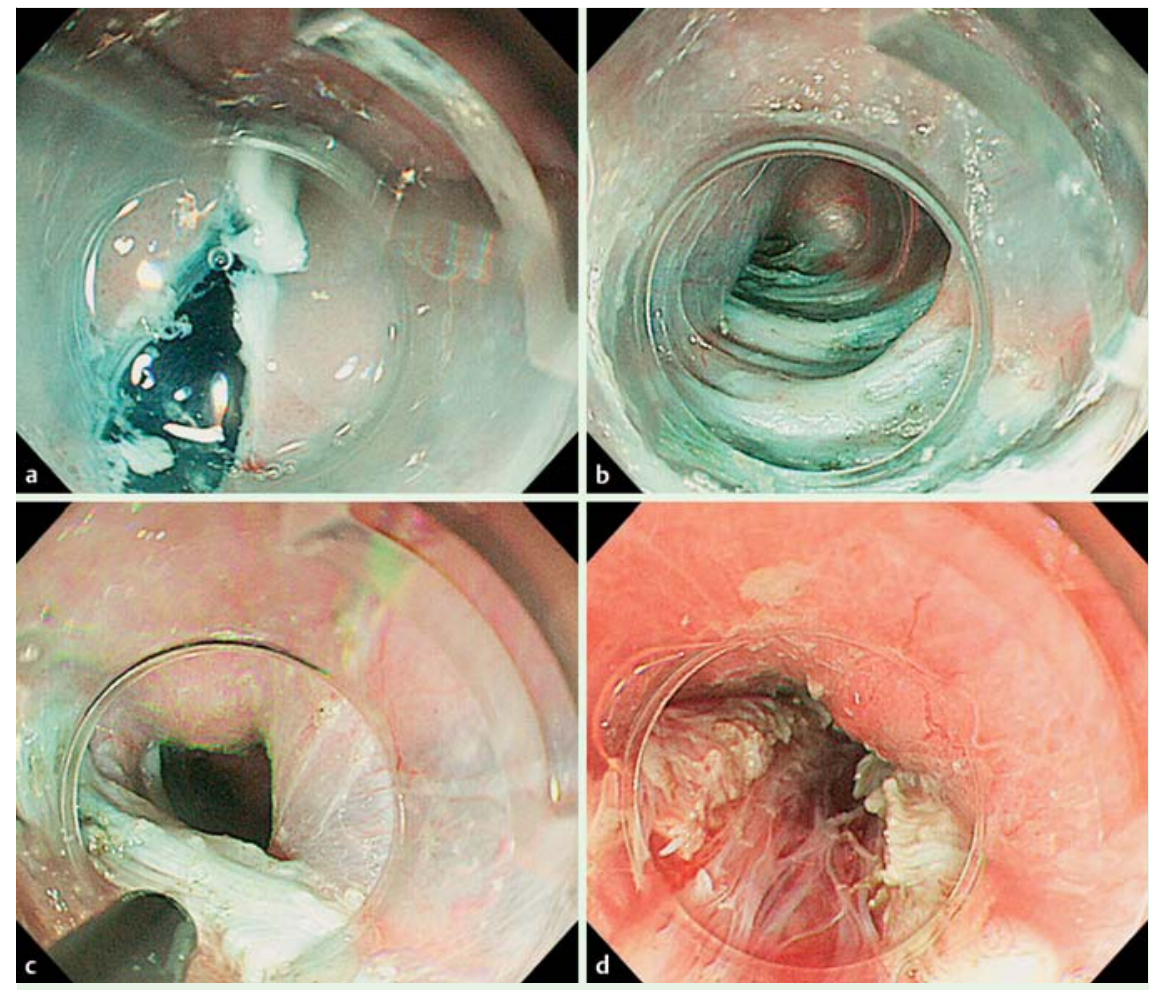

Fig. 4 Peroral endoscopic myotomy. a Mucosal incision site at the 5-o'clock position in the mid esophagus. $\mathbf{b}$ Endoscopic view of the submucosal tunnel. c Selective myotomy of circular muscle fibers. d After myotomy.

other hand, POEM is a minimally invasive procedure with an approach from the esophageal lumen that is not complicated by the effects of previous surgery [4]. Recently, myotomy has often been performed at an anterior (11- or 2-o'clock) position or a posterior (5-o'clock) position because there is less risk for gastroesophageal reflux [5]. However, the 11- and 2-o'clock positions should be avoided in patients who have severe fibrosis and staples from previous surgery. We successfully treated this patient with a myotomy at the 5-o'clock position. When a patient with achalasia and a distal gastrectomy is treated with POEM, selecting the appropriate myotomy position is of the utmost importance.

\section{Endoscopy_UCTN_Code_TTT_1AO_2AN}

\section{Competing interests: None}

\section{References}

\section{Shinwa Tanaka , Fumiaki Kawara', Haruhiro Inoue ${ }^{2}$, Manabu Kurosawa ${ }^{3}$, Takashi Toyonaga', Takeshi Azuma ${ }^{3}$}

${ }^{1}$ Department of Endoscopy, Kobe University Hospital, Kobe, Japan

2 Digestive Disease Center, Showa University Koto-Toyosu Hospital, Tokyo, Japan ${ }^{3}$ Division of Gastroenterology, Department of Internal Medicine, Graduate School of Medicine, Kobe University, Kobe, Japan

1 Pandolfino JE, Gawron AJ. Achalasia: a systematic review. JAMA 2015: 12; $1841-1852$

2 Ramos AC, Murakami A, Lanzarini EG et al. Achalasia and laparoscopic gastric bypass. Surg Obes Relat Dis 2009; 5: 132-134

3 Chapman R, Rotundo A, Carter N et al. Laparoscopic Heller's myotomy for achalasia after gastric bypass: a case report. Int J Surg Case Rep 2013; 4: 396-398

4 Inoue H, Minami H, Kobayashi Yet al. Peroral endoscopic myotomy (POEM) for esophageal achalasia. Endoscopy 2010; 42: 265 271

5 Bechara R, Ikeda H, Inoue H. Peroral endoscopic myotomy: an evolving treatment for achalasia. Nat Rev Gastroenterol Hepatol 2015; 12 : 410-426

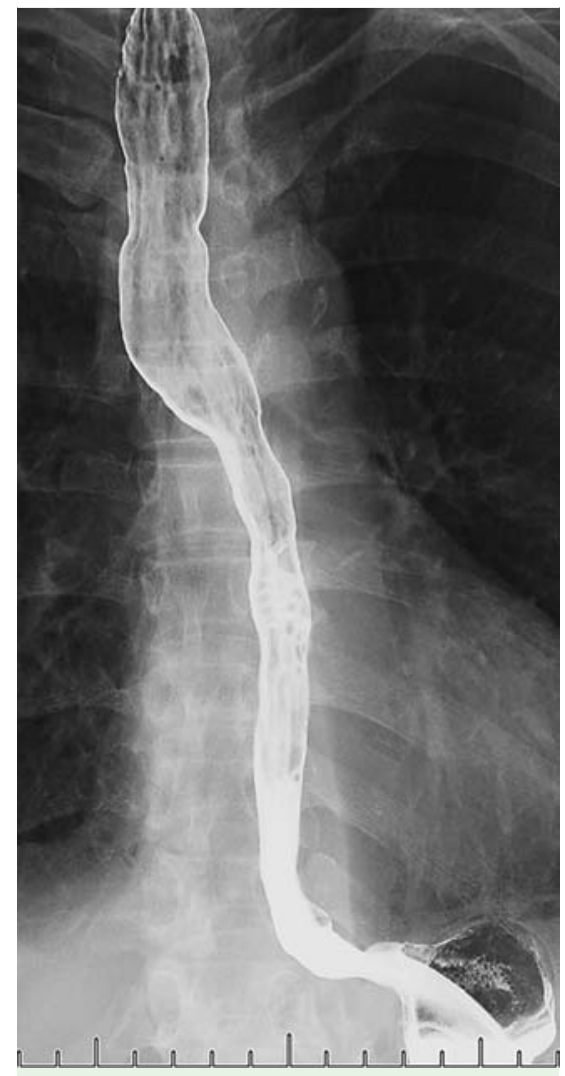

Fig. 5 Esophagogram obtained on postoperative day 1 revealing adequate passage of contrast into the remaining portion of the stomach.

Bibliography

DOI http://dx.doi.org/

10.1055/s-0034-1393229

Endoscopy 2015; 47: E511-E512

(c) Georg Thieme Verlag KG

Stuttgart · New York

ISSN 0013-726X

Corresponding author

Shinwa Tanaka, MD

Department of Endoscopy

Kobe University Hospital

7-5-1 Chu-o-ku

Kusunoki-Cho

Kobe

Hyogo 650-0017

Japan

Fax: +81-78-382-6309

tanakas@med.kobe-u.ac.jp 